\title{
Diversidades sexuales e identidades de género: entre la aceptación y el reconocimiento. Instituciones de Educación Superior (IES) ${ }^{*}$
}

DOI: https://doi.org/10.18046/recs.i31.3261

\author{
Sexual Diversity and Gender Identity: Between Acceptance \\ and Recognition. Higher Education Institutions (HEI)
}

\author{
Alba Nubia Rodríguez-Pizarro** \\ Universidad del Valle (Cali, Colombia)
}

Janet Rivera-Crespo***

Universidad del Valle (Cali, Colombia)

\footnotetext{
* Artículo de investigación resultado del proyecto de investigación Aportes a la política de género de la Universidad del Valle para la construcción de una sociedad en paz, financiado por Colciencias en la convocatoria 740 de 2016. El proyecto fue ejecutado durante el período 2016-2018. Artículo de investigación recibido el 16.11.2018 y aceptado el 20.12.2019.

** Doctora en Ciencias Políticas y Sociología por la Universidad Complutense de Madrid (España). Magíster en Sociología de la Universidad del Valle (Colombia). Antropóloga de la Universidad del Cauca (Colombia). Profesora titular de la Escuela de Trabajo Social y Desarrollo Humano de la Universidad del Valle (Colombia). Correo electrónico: alba. rodriguez@correounivalle.edu.co ORCID: https://orcid.org/oooo-ooo3-1055-9636

*** Especialista en Gerencia Social de la Pontificia Universidad Javeriana, sede Cali (Colombia). Especialista en Teorías, Técnicas y Métodos de Investigación Social y socióloga de la Universidad del Valle (Colombia). Estudiante-investigadora del Doctorado de Humanidades, Línea Estudios de Género, de la Universidad del Valle (Colombia). Correo electrónico: janet.rivera@correounivalle.edu.co ORCID: https://orcid.org/oooo-ooo2-1389-3077
} 


\section{Cómo citar/How to cite}

Rodríguez-Pizarro, Alba Nubia; Rivera-Crespo, Janet (2020). Diversidades sexuales y de identidades género: entre la aceptación y el reconocimiento. Instituciones de Educación Superior (IES). Revista CS, 31, 327-357. https://doi.org/10.18046/recs.i31.3261 


\section{Resumen}

Este artículo tiene como propósito reflexionar sobre la lógica heteronormativa ${ }^{1}$ que transversaliza las relaciones entre los diferentes actores que conforman la comunidad universitaria, cuya incongruencia se expresa en la práctica cotidiana, y plantear la necesidad de reconocer y resignificar las relaciones con personas con orientaciones sexuales e identidades de género no hegemónicas como un aspecto fundamental para el logro de la justicia de género, que implica a las personas, a las instituciones de educación y a la sociedad en general. Si bien el énfasis se hace en las orientaciones sexuales e identidades de género no hegemónicas, se asume una perspectiva epistemológica compleja en la que género y sexualidad se entrelazan con otros patrones de dominación como la etnicidad, la raza, la clase social, la edad, entre otros.

\section{PALABRAS CLAVE:}

orientación sexual, identidad de género no hegemónica, heteronormatividad, discriminación, reconocimiento

The purpose of this paper is to reflect on the heteronormative logic that mainstreams relationships between the different actors constituting the university community (whose incongruity is expressed in everyday practice) and to raise the need to recognize and re-signify the relationships with people with non-hegemonic sexual orientations and gender identities as a fundamental aspect for achieving gender justice. Which involves people, education institutions, and society in general. Although the emphasis is placed on non-hegemonic sexual orientations and gender identities, a complex epistemological perspective is assumed in which gender and sexuality are intertwined with other patterns of domination such as ethnicity, race, social class, age, among other.

\section{KEYWORDS:}

Sexual Orientation, Non-Hegemonic Gender Identity, Heteronormativity, Discrimination, Recognition

\footnotetext{
1. De acuerdo con el Instituto Nacional contra la Discriminación, la Xenofobia y el Racismo (INADI, 2016: 108), "se habla de heteronormatividad, heterosexismo o heterosexualidad obligatoria en referencia al paradigma que presenta a la heterosexualidad como natural y necesaria para el funcionamiento de la sociedad, y como el único modelo válido de relación sexual, afectiva y de parentesco. Se sostiene y reproduce a partir de instituciones que legitiman y privilegian la heterosexualidad y de variados mecanismos sociales que incluyen la invisibilización, exclusión y persecución de todas las manifestaciones que no se adecuen a él”.
} 



\section{Introducción}

El estudio Aportes a la política de género de la Universidad del Valle para la construcción de una sociedad en paz, realizado por los grupos de investigación que integran al Centro de Investigaciones Estudios de Género, Mujer y Sociedad ${ }^{2}$, contribuyó a la construcción de los fundamentos para la formulación de la Política Pública de Género para la Universidad del Valle, que como Institución de Educación Superior, debe contribuir a generar procesos sociales y culturales que promuevan la consolidación de la sociedad colombiana, como una sociedad incluyente, equitativa y libre de discriminaciones, condiciones fundamentales para lograr una sociedad en paz.

La investigación se realizó con la participación de los distintos actores de la comunidad académica: profesorado, alumnado, personal administrativo y trabajadoras(es) de la universidad. No obstante, en la escritura del presente artículo, privilegiamos las voces del profesorado y el estudiantado, tanto de las sedes de Cali como de las nueve sedes regionales que componen esta universidad.

La estrategia metodológica utilizada en el proyecto combinó métodos cuantitativos con diseño de agregados y encuestas que se realizaron a todos los estamentos de la comunidad universitaria, y métodos cualitativos en los que se privilegiaron las entrevistas y los grupos focales con profesorado y estudiantado. Se realizaron 18 grupos focales en las sedes regionales y 8 en Cali, con estudiantes de diferentes programas académicos, en los que participaron personas que se identificaron con orientaciones sexuales e identidades de género no hegemónicas como también personas que se reconocieron como heterosexuales y con identidades étnico-raciales afro y mestizas. De igual manera, se realizaron veinte entrevistas al profesorado y directivas.

En este documento se hace alusión a los datos cualitativos obtenidos a partir de las dos técnicas mencionadas, las cuales permitieron profundizar en la comprensión de las experiencias de invisibilidad y participación condicionada en el ámbito universitario de las personas con orientaciones sexuales e identidades de género no hegemónicas. Al mismo tiempo, permitieron desvelar los mecanismos mediante los cuales la heteronormatividad, como lógica hegemónica de la sociedad y en este caso particular de una Institución de Educación Superior, favorece procesos de exclusión, pese a que algunos actores asumen el espacio de la universidad como abierto

2. Grupos: Sujetos y Acciones Colectivas (Facultad de Humanidades), Estudios Étnico Raciales (Facultad de Ciencias Sociales y Económicas), Género y Política (Facultad de Humanidades, Facultad de Ciencias Sociales y Económicas e Instituto de Psicología) y Educación Popular (Instituto de Educación y Pedagogía), con la colaboración de la profesora Martha Zapata de la Universidad de Berlín y Sara Motta de la Universidad de Nottingham. 
y propicio para manifestar las diferencias y las diversidades. En este contexto, la heterosexualidad, de manera implícita o explícita, otorga un estatuto de superioridad o privilegio a todos los que se adecuan a tal orientación, a la vez que legitima la discriminación de las personas con orientaciones sexuales y de géneros diversas.

En el mundo, y también en Colombia, presenciamos un cambio favorable y progresivo, que cada vez más visibiliza y reconoce a las personas con orientaciones sexuales e identidades de género no hegemónicas. De acuerdo con el Fondo de Población de Naciones Unidas (UNFPA, 2012), este cambio se debe a la lucha continua y a las acciones colectivas emprendidas por esta población para el reconocimiento y efectivo cumplimiento de sus derechos. Transformaciones que han sido estimuladas, en parte, por la resignificación tanto de las libertades individuales, como de los derechos humanos, lo cual ha posibilitado la legitimación de discursos de apertura y de inclusión. Lo anterior se refleja en el avance legislativo en diferentes países, en general, y en Colombia, en particular, y en la ganancia de espacios sociales, políticos y económicos por parte de las personas LGTBI. Al respecto, Martínez (2018) señala la importancia de hacer rupturas epistemológicas que permitan interpretar y analizar estas realidades más allá de las reivindicaciones y conquistas legales, para lo cual propone hacer una lectura de las movilizaciones LGBTI, queer, trans y transfeministas como procesos de construcción de las subjetividades políticas a partir de las disidencias sexuales y corporales, entendidas como procesos subjetivos de mutación y resistencia a procesos heteronormativos de producción del cuerpo y de la sexualidad. Lo anterior permite visualizar los distintos matices de estas construcciones y comprender que el límite del reconocimiento no puede quedar establecido en el marco legislativo y normativo existente en los diferentes países. Esto con el propósito de que los mecanismos de discriminación que se expresan en la cotidianidad y se disfrazan en la normalidad no pongan en entredicho el pleno reconocimiento de estas personas.

Las Instituciones de Educación Superior no son ajenas a lo que algunos estudiosos han llamado como binarismo de género o bigenerismo, de acuerdo con Gilbert (2011), para referirse a esa lógica de opuestos, que concibe hombre y mujer como las únicas categorías en que se dividen los seres sexuados, y que les califica como imperfectos en tanto más se alejen del patrón bigenerista. Desde esa perspectiva, estas categorías son entendidas como complementarias y excluyentes entre sí. Contrario a esta concepción, observamos que las prácticas cotidianas y las vivencias de las personas reflejan la inestabilidad y la fluidez que caracteriza a los seres humanos. Tanto el binarismo de género como la heteronormatividad evidencian un sistema jerárquico de relaciones sociales que en la universidad se expresa a través de prácticas y comportamientos de aceptación y a la vez de rechazo, discriminación 
y violencias que ocurren contra las personas con orientación sexual e identidades de género no hegemónicas que integran la comunidad académica.

Los estudios sobre la orientación sexual e identidad de género diversa en las universidades son recientes, y se relacionan con el avance de procesos de movilización, promovidos por esta población, a partir de los años noventa. El interés por el tema surge en las universidades de la mano de los estudios de género y de masculinidades, especialmente de la homosexualidad masculina. De manera tímida, en la década de los noventa, pero con mayor determinación a partir de los primeros años de la década del 200o. Es así como en el período 1994-2010, el interés de la comunidad académica internacional en este tema giró en torno a la comprensión de la vivencia de la homosexualidad en la cotidianidad universitaria, a la identificación de sus demandas y a la propuesta de políticas institucionales y metodologías de intervención dirigidas a esta población en los campus universitarios.

En América Latina, los intereses académicos sobre el tema profundizaron en las percepciones y representaciones del estudiantado sobre las personas con orientación homosexual, la convivencia en el espacio universitario y las legislaciones que garantizaban sus derechos. Entre 1995 y 2010 se realizaron estudios en la ciudad de Cali sobre la homosexualidad femenina y masculina, las representaciones de la homosexualidad en los medios de comunicación local, la evaluación de la implementación de las leyes que garantizaban los derechos de esta población en el Valle del Cauca, y los llamados crímenes de odio en la ciudad (Uribe, 2012).

Otras investigaciones realizadas en Colombia son las de Arango (2017); Guerrero y Zambrano (2013); López, Martínez, Negrete y Paternina (2016), y Zambrano y Escalante (2013), que se enfocan en el estudio de la discriminación hacia personas con orientación sexual e identidad de género no hegemónica en las universidades. Se destaca el interés de algunos estudios por la comprensión de la homofobia, lesbofobia y transfobia, cruzadas con marcadores de género y raza en estudiantes que se están formando en áreas de la salud (Arango, 2017; Campo; Oviedo; Herazo, 2017; Guerrero; Zambrano, 2013; (López; Martínez; Negrette; Patermina, 2016; Zambrano; Escalante, 2013).

Fernández, Hernández y Paniagua (2013), en su estudio sobre la violencia de género en la Universidad de Antioquia, identificaron que la población estudiantil con orientación sexual diferente a la heterosexual manifestó haber sentido acoso sexual, agresión verbal y psicológica, gestos obscenos, burlas y piropos obscenos, maltrato y sanción por su orientación sexual, los cuales interpretaron como expresiones homofóbicas que actúan mediante mecanismos de ignorancia, particularización y calificación moral. 
Vergel, Martínez y Zafra (2016), en una investigación sobre los factores que inciden en el bullying en estudiantes de trece Instituciones de Educación Superior de Norte de Santander, observaron quiénes son más vulnerables a este tipo de prácticas y por qué. En los resultados de su estudio encontraron que el bullying es más frecuente en las mujeres, estudiantes jóvenes y homosexuales. Y entre los factores que inciden para que las(os) estudiantes sean víctimas de bullying están: la orientación sexual, la identidad de género, la apariencia del cuerpo, la etnia, el estado anímico, la religión y la región de procedencia.

La revisión-no exhaustiva- de estos estudios permite señalar que la inclusión de aspectos como la orientación sexual e identidad de género en la agenda educativa nacional es reciente y escasa, pero cada vez hay un mayor interés por analizar qué sucede en las Instituciones de Educación Superior con esos nuevos sujetos sociales antes totalmente marginados.

Este escrito se inscribe en el horizonte de los trabajos mencionados con el propósito de presentar esa lógica de opuestos que se manifiesta en el ámbito de la universidad: por un lado, la aceptación, el reconocimiento de algunos derechos, y por otro, los comportamientos excluyentes e inferiorizantes hacia personas con orientación sexual e identidad de género no hegemónica.

\section{Claves teóricas y conceptuales}

Asumimos que tanto el género como la sexualidad son construcciones históricas y culturales, y que el dinamismo de las relaciones sociales somete sus categorías, nociones y valoraciones a una permanente confrontación y redefinición, que invita a la deconstrucción de modelos únicos que jerarquizan moral y políticamente las sexualidades (Moore, 1991). La perspectiva de género permite desnaturalizar dichas categorías por su capacidad explicativa, en tanto reconoce otros actores que también son víctimas de discriminación por no asumir estereotipos tradicionales de masculinidad y feminidad, definidos socialmente. En este sentido, no solo describe el fenómeno, sino que explica y hace visible las cambiantes identidades de género en las sociedades contemporáneas, así como la variedad de formas de opresión que se basan en los estereotipos, prejuicios y discriminaciones basadas en género (Serret, 2011). Adicionalmente, su carácter relacional, nos permite entender que las representaciones, valoraciones y normas adjudicadas a lo femenino y lo masculino constituyen modos de significar relaciones de poder y estructurar las relaciones sociales, así como la subjetividad individual (Scott, 2008).

$\mathrm{Al}$ igual que el género, la sexualidad se asume como una construcción histórica, social y cultural que abarca orientación sexual, erotismo, placer, intimidad 
y reproducción, y está en permanente redefinición y disputa (Instituto Nacional Contra la Discriminación, Xenofobia y el Racismo [INADI], 2016). De acuerdo con la Organización Mundial de la Salud (OMS, 2015), la sexualidad se entiende como un aspecto central del ser humano a lo largo de su ciclo vital. Sexualidad y género se entrecruzan con otros marcadores de discriminación como la pertenencia étnico-racial, la clase social, la edad, entre otras, para profundizar la estigmatización y la exclusión (Figari, 2008).

Como advierten Curiel (2011), Kimmel (1997), Lugones (2008) y Viveros (2016), todas estas articulaciones configuran un entramado de relaciones asimétricas de poder en contextos singulares, que denominan interseccionalidad, entendida, en términos de Munévar (2013: 14),

como la base del reconocimiento de las diferencias y las diferenciaciones para analizar las posiciones subjetivas de enunciación, comprender las formas de producción del poder, el privilegio y la marginalización de las personas a través de los arreglos identitarios interseccionados a partir de las múltiples categorías sociales subyacentes. Sin duda, es una convocatoria epistémica para traspasar los límites del pensar occidental binario, hegemónico o esencialista, sobre todo porque, al comprender el género en su expresión articulada e inseparable de otras categorías sociales, relaciona el poder como vía para tomar distancia de la visión impuesta que considera a las mujeres como un grupo homogéneo y devela las razones por las cuales muchas situaciones vividas por mujeres y hombres de todas las condiciones, quedan invisibles y opacadas.

Si género y sexualidad son dos categorías construidas social y culturalmente, e interrelacionadas, se entiende que la identidad de género y la orientación sexual son independientes del sexo asignado con base en las diferenciaciones anatómicas de los seres humanos. La primera se refiere a la vivencia interna e individual del género, se relaciona con cómo se siente y se vive el cuerpo desde la experiencia personal y cómo se lleva al ámbito público (Barrancos, 2006), de ahí que implique cambios en la apariencia corporal a través de la forma de vestir, de hablar, de los modales, entre otros. La segunda se ha definido como la capacidad de cada persona de sentir una profunda atracción emocional, afectiva y sexual por personas de un género diferente al suyo, de su mismo género o de más de un género, así como a la capacidad de mantener relaciones íntimas y sexuales (Comisión Internacional de Juristas [CIJ], 2009).

La orientación sexual tensiona la noción acerca de la existencia de una forma de ejercer la sexualidad y los afectos, haciendo visible la existencia de otras maneras de expresarlos. Incluye la heterosexualidad, la homosexualidad y la bisexualidad; de esta manera se aleja de la concepción binaria y heteronormativa prevaleciente 
en la sociedad local, regional y nacional. Entendemos las diversidades de orientaciones sexuales y de identidades de género, de acuerdo con el INADI (2016), como multiplicidad de prácticas y manifestaciones emocionales, afectivas y sexuales en una cultura dada.

Por otra parte, algunos de los estudios revisados (Pecheny; Figari; Jones, 2008; Cáceres; Rosasco, 200o; Castelar; Lozano, 2018) coinciden en el debate sobre cómo nombrar las diversidades y la intencionalidad que encierra hacerlo de una manera o de otra. Compartimos con estos autores y autoras la propuesta de un acercamiento que exalta la emergencia de nuevos sujetos sexuales, asociados a procesos de organización colectiva y a una noción de ciudadanía sexual. Se asume a las personas con orientación sexual y de género no hegemónica como sujetos sexuales con derechos, con capacidad de actuación y de decisión, y no como víctimas, lugar desde el cual se despolitizan, en tanto impide que se constituyan como sujetos colectivos.

Finalmente, es importante señalar que, de acuerdo con la OMS (2015), los aspectos negativos de la discriminación hacia las personas con sexualidad no hegemónica, afectan no solo la salud de quienes comparten características que socialmente las marcan como inaceptables (ser VIH positivo, tener relaciones sexuales con personas del mismo sexo, ser un adolescente sexualmente activo, trabajador(a) sexual, transgénero, intersexual o cualquier combinación de estos), sino que se constituyen en casos de violación de los derechos humanos como el derecho a la salud, a la vida, a la igualdad, a la no discriminación, a la privacidad, y el estar libre de tratos inhumanos y degradantes, entre otros. En consecuencia, el reconocimiento de las personas con orientación sexual y de género no hegemónica implica una deconstrucción del binarismo de género y de la heteronormatividad que instituye la heterosexualidad como norma y permea todas las relaciones sociales en los distintos ámbitos de la comunidad universitaria, dando lugar a la discriminación; la cual tiene que ver con las representaciones socioculturales de la normalidad y con el poder de ciertos grupos para atribuírsela.

\section{Análisis de resultados sobre diversidades sexuales e identidades de género: procesos, debates e inclusión en la Universidad del Valle}

De acuerdo con lo expresado en las entrevistas realizadas en la investigación, durante la década de los ochenta en la Universidad del Valle los estudios sobre las mujeres tenían un lugar preponderante; posteriormente, en los años noventa se incorporó la perspectiva de género, la cual ya no solo privilegiaba los estudios sobre las mu- 
jeres, sino también sobre masculinidades, que si bien en la universidad han estado presentes, su desarrollo como campo de análisis, a la fecha, no ha sido protagónico.

La Constitución de Colombia de 1991, en la que se reconoce la diversidad étnica y cultural de la nación, marca un hito muy importante para el reconocimiento de las diversidades étnico-raciales, las orientaciones sexuales e identidades de género no hegemónicas en la sociedad colombiana ${ }^{3}$. Es así como en la década del 200o, algunas(os) académicas(os) pusieron en la agenda de reflexión y discusión las diversidades en orientación sexual y de identidades de género. No obstante, el énfasis se hizo en la homosexualidad masculina, como se expresó en una de las entrevistas realizadas:

Los estudios y el tema diversidad de género son posteriores a los de género, hace diez o quince años, esa cuestión de la diversidad sexual empieza con el trabajo de Carlos Arango, con personas que no están dentro de la perspectiva de género, como Octavio Giraldo Neira quien empezó esto en psicología, pero no desde perspectiva de género, hubo que esperar que la gente empezara a leer a Judith Butler y eso lo escribe ella después y tiempo más tarde, aparece. En la universidad la diversidad sexual se planteaba más desde la masculinidad, que desde la feminidad, las mujeres estaban invisibles respecto al tema. (Docente, Facultad de Humanidades, sede Cali, comunicación personal, 2017) ${ }^{4}$

Por otra parte, desde mediados de los años ochenta, en la Facultad de Salud, emergieron los primeros trabajos sobre sexualidades no hegemónicas. En los noventa, la universidad ya contaba con iniciativas individuales de docentes trabajando en el reconocimiento de la diversidad de orientaciones sexuales a nivel nacional y latinoamericano, que se consolidaron en el Programa sobre Sexualidad Humana que nació de la confluencia entre una profesora de la Facultad de Salud y profesores del hoy Instituto de Psicología. Adicionalmente, la Facultad de Salud cuenta desde entonces con una docente pionera que continúa trabajando el tema desde el campo de la investigación y de la intervención, especialmente realizando acompañamientoy

3. La primera intervención normativa por parte de la universidad con este propósito se refiere a la cuota de excepción étnica. En 1998, se creó la condición de excepción para indígenas, y en la década del 2000, específicamente en 2005, se inició el proyecto Universidady culturas, que ya no solo se ocupa del ingreso, sino que se dirige a buscar la permanencia de las poblaciones étnico-raciales.

4. Esta tendencia coincide con un estudio realizado en Colombia por Serrano y Viveros (2006), que señala cómo en los medios de comunicación del año 2003 se dio una vasta producción de telenovelas y programas de opinión con imágenes de hombres homosexuales, en las cuales las mujeres lesbianas también estaban invisibilizadas. 
apoyo integral a algunos(as) estudiantes que decidieron hacer el tránsito de un género a otro, e incluso cambiar de sexo, como ella lo expresó en la entrevista realizada:

Se me empezaron a acercar los primeros estudiantes, y llegaron personas que decían no saber qué son (...) los veía en consulta, los veo sola o con los residentes, pero no les cobro (...) trabajo con personas trans hace veinte años, muchos ya se han graduado. Por ejemplo, Mario entró como hombre y salió como mujer, Mariana, que se va a graduar ahora, les he acompañado en el proceso (...) la gente no sabe. A un paciente trans hombre, le decía el endocrinólogo que tenía que seguir siendo mujer, que poniéndose vestido dos semanas se le quitaba eso. (Docente, Facultad de Salud, sede Cali, comunicación personal, 2017)

A pesar de lo anteriormente señalado, estas iniciativas de algunos(as) docentes no permearon la institucionalidad universitaria con un enfoque más abierto y comprometido. Es así como en la década del 2000, las diversidades sexuales y de género pasaron, en la universidad, de una preocupación académica minoritaria tardía al cuestionamiento por la presencia, participación y visibilización de una población que hacía parte de la comunidad universitaria y que no había sido visibilizada ni reconocida. En este proceso, influyó la movilización nacional de los colectivos LGTBI ${ }^{5}$, así como la constituyente social promovida por el entonces gobernador del Valle del Cauca, Angelino Garzón, quien, en alianza con un grupo de personas de la universidad, apoyó la iniciativa de visibilizar la existencia de personas con orientaciones sexuales e identidades de género no hegemónicas, a través de la realización de un foro público sobre diversidades, en el año 2006:

Fue una coyuntura especial, no es resultado de discusiones previas, esta discusión se instaló en la universidad a partir del foro. El organizador trabajó un año solo, recogiendo firmas para tener suficiente respaldo para convocar a un foro público, de oficina en oficina, profesor por profesor. (Docente, Facultad de Ciencias Sociales y Económicas, sede Cali, comunicación personal, 2017)

El debate sobre este tema se instauró en la Universidad del Valle, con el foro sobre Diversidades Sexuales y de Género, que logró el apoyo de varios centros de investigación de la universidad (CIDSE, Centro de Estudios de Género, Mujer y Sociedad, entre otros) y contó con la participación de la rectoría. Fue importante la sensibilización del personal de vigilancia con respecto a este sector poblacional de la 
comunidad universitaria. En una de las entrevistas, se exalta la importancia del evento para dimensionar la visibilidad y participación de esta población en la universidad:

El evento representó un hito histórico en la universidad. Por primera vez más de 200 personas interesadas en esta problemática se reunieron, se reconocieron mutuamente y formularon las primeras reflexiones y propuestas para su visibilización y participación en la vida universitaria. Las directivas universitarias acogieron sin reservas la propuesta y realización del foro. La jornada implicó un aprendizaje de construcción de democracia y pluralismo para todos los estamentos universitarios. El sector de vigilantes ubicados en las porterías de la universidad y en los edificios recibieron instrucciones para permitir la entrada sin ningún tipo de discriminación a los participantes y para proteger la integridad personal, psíquica y física de los asistentes. La realización de este foro público se constituyó así en un ejercicio de aprendizaje alrededor de la visibilización y el respeto de las diferencias sexuales y de identidad. (Docente, Instituto de Psicología, comunicación personal, 2017)

Al respecto, la rectoría de ese entonces manifestó la necesidad de crear condiciones en la universidad para la organización y realización del foro:

Consideramos estratégicamente oportuno y saludable, que se realice un foro público sobre las diversidades sexuales en la universidad, y que busquemos la creación de condiciones actitudinales y valorativas para que se promueva la participación de la comunidad universitaria conjuntamente con los gais, lesbianas, bisexuales y transgeneristas, en el análisis de la situación sobre las libertades eróticas y afectivas, así como también que se reflexione sobre el problema de la visibilización y caracterización del sector LGBTI en el contexto de una cultura estigmatizante, de tal manera que se elaboren reflexiones y propuestas que lleguen a quedar incluidas en la constituyente social del Valle del Cauca. Igualmente consideramos que esta es una excelente oportunidad para que se promueva la organización y participación del sector LGBTI de Univalle, de tal manera que se dé el reconocimiento y la acción colectiva, se desarrollen estrategias de comunicación, intercambio de experiencias, amistades, afectos, placeres y apoyo mutuo, a la vez que se desarrollen actividades culturales y educativas dirigidas a toda la comunidad universitaria. (Arango, 2006: 8)

Aunque las entrevistas realizadas nos llevan a plantear como punto de inflexión el año 2005 y la realización del foro, no podemos desconocer el grupo Colectivo de Diversidades Sexuales de la Universidad del Valle, referenciado por Uribe (2012) en el estudio Los grupos sociales de los sexualmente diversos en contexto universitario, donde se refiere ampliamente a la importancia de este grupo conformado por estudiantes y profesorado, que pese a la itinerancia y participación temporal de sus integrantes, se 
constituyó con el propósito de discutir el tema de la diversidad sexual y propiciar nuevos espacios de inclusión y de encuentro para las(os) jóvenes.

Aunque en la práctica sigan existiendo limitaciones para su pleno reconocimiento, los colectivos de diversidades y el foro abrieron el debate que aún no se retoma con la centralidad que, desde nuestro punto de vista, debía tener. No obstante, es innegable el efecto de estos y de la movilización social en la progresiva apertura de la comunidad universitaria. En sus voces:

Llegan como pareja: mujer-mujer u hombre-hombre. Consideran que ha habido un cambio en este sentido porque hace algunos años no era tolerado y se tenía mucha discriminación (burlas, chistes, mofas); el cambio generacional y las redes sociales permiten una mirada amplia y sin prejuicios. (Docente, sede regional Tuluá, acta del grupo focal, 2018)

Los esfuerzos concretos por parte del estudiantado y el profesorado han llevado a que, a partir del año 2017, la universidad cuente con iniciativas como Campus Diverso, el cual hace acompañamiento estudiantil a personas con orientaciones sexuales diversas e identidades de género no hegemónicas, así como a personas con VIH. Un programa con enfoque interseccional que involucra medicina familiar, psicología y trabajo social, donde actualmente participan veinte estudiantes que llegan al programa por voluntad propia, remitidos por amigos o por líneas de atención como el $106^{6}$.

Desde el nivel normativo institucional de la universidad (Acuerdo oo1, enero 29 de 2002 del Consejo Superior7; Plan Estratégico de Desarrollo 2005-2015; ; Resolución núm. 2516 de 2010 de Rectoría, y Resolución 055 de $2015^{9}$ ), se hace un esfuerzo por acercarse a la tendencia internacional y nacional que busca el reconocimiento de sujetos sociales históricamente marginados como son los gais, lesbianas, trans, afrodescendientes e indígena. Como lo expresa Ochy Curiel (2012:15): "Nuevos sujetos que no figuraban en el escenario público porque no correspondían al paradigma de la modernidad, o sea, el sujeto que es hombre, blanco, heterosexual y con privilegios de clase". Sin embargo, mientras la universidad, por un lado, avanza al reconocer los derechos de una población atravesada por marcadas diferencias, desde su práctica institucional, algunos integrantes de la comunidad universitaria se resisten de múlti-

\footnotetext{
6. Datos obtenidos en entrevista realizada a profesional de apoyo del programa.

7. Ver http://secretariageneral.univalle.edu.co/consejo-superior/acuerdos/20O2/acu-oo1.pdf

8. Ver http://www.univalle.edu.co/component/k2/plan-estrategico-de-desarrollo-2005-2015

9. Ver http://secretariageneral.univalle.edu.co/rectoria/resoluciones/2010/r-2516.pdf
} 
ples formas a reconfigurar su cultura heteronormativa y heterosexista, exponiendo a estas personas a experiencias dolorosas. Así lo ilustra el testimonio de una docente:

Si bien, no hay ninguna normatividad institucional que prohíba expresiones de las diversidades, especialmente las de orientación sexual, estas personas no se expresan con libertad, porque hay burlas, chistes, comentarios y se comportan delante de la profesora de un modo y cuando ella no está se hacen de otro modo. (Docente, sede regional Santander de Quilichao, acta del grupo focal, 2018)

Al mismo tiempo que se configura un discurso de tolerancia y de aceptación de las personas con orientación sexual y de género diversa, emerge una paradoja en la universidad, que se expresa a través de prácticas y señalamientos cotidianos como burlas, chistes o ciberhostigamiento hacia las personas con orientaciones sexuales e identidades de género no hegemónicas. De esta manera, por un lado, se acepta y valora como normal la presencia y la participación de estas personas en la universidad, y por otro, son discriminadas y violentadas. En la base de estas prácticas incoherentes por parte de la comunidad universitaria, está la idea de que la heterosexualidad es el único modelo válido de relación sexual y afectiva, la cual configura el núcleo de prácticas sociales discriminatorias hacia todo aquello que no se corresponda con la heteronormatividad, también un orden de género que actúa en lo institucional, en las relaciones y en el ámbito simbólico, el cual se fundamenta en el binarismo. Así, se invisibiliza y se vulnera el reconocimiento de las personas con orientación sexual y de género no hegemónica (Rich, 1980; Wittig, 1992).

\section{Mecanismos de exclusión de las personas con orientaciones sexuales e identidades de género no hegemónicas en la IES}

A pesar de los progresivos -pero aún insuficientes- cambios de actitud por parte de la comunidad universitaria, persisten mecanismos de exclusión que se expresan en diferentes ámbitos del contexto universitario y afectan el reconocimiento de las personas con orientaciones sexuales e identidades de género no normativas. En este sentido, tienen un gran peso en la dinámica cotidiana universitaria de interacciones cargadas de comportamientos y expresiones que descalifican y que influyen para que estas personas sean agredidas, señaladas y se conviertan en objeto de hostigamiento soterrado, maltrato, aislamiento y bromas. Este conjunto de prácticas y comportamientos cotidianos que ocurren en diferentes escenarios (salones de clase, baños, cafeterías, espacios deportivos, entre otros) se manifiesta al toparse con las 
orientaciones sexuales e identidades de género no hegemónicas en la universidad, basándose en una lógica heteronormativa que circula por los diferentes espacios y permea las relaciones, constituyendo un ambiente incoherente con los principios y valores que inspiran la educación superior y condiciones de vida digna.

Si bien, inicialmente hay una referencia general a los mecanismos de discriminación, exclusión y no reconocimiento, es fundamental diferenciar cómo operan estos ante las personas con orientaciones sexuales diversas o con identidades de género no hegemónicas. En este sentido, es un imperativo de primer orden diferenciar las expresiones de discriminación, burla y no reconocimiento que se expresan con personas con orientación sexual diversa: gais, lesbianas, bisexuales, pansexuales; y las que se expresan como personas con identidades de género no hegemónicas: transgénero y transexuales. Como señalan estudios similares, "el nivel de prejuicio y distancia entre hombres heterosexuales y gais es mayor que entre mujeres y lesbianas" (Toro-Alfonso; Varas, 2004, como se citó en Uribe, 2012: 29).

Para comprender de manera diferenciada las agresiones que se ejercen contra las personas trans, es fundamental aludir al orden de género imperante en la sociedad colombiana, en general, y en la sociedad local, en particular, el cual se fundamenta en una lógica binaria, sustentada en que el género tiene su génesis en una diferenciación natural que existe con anterioridad a la vida social, como es el sexo, lo cual hace que este sea inmutable, asocial y transhistórico. De esta manera, la construcción de las identidades y las expresiones de género se asumen como esenciales y no como construcciones sociales, culturales e históricas y por lo tanto cambiantes. En el ámbito universitario, pese a la normatividad y a la construcción de estrategias de apertura a los procesos conjuntos agenciados por los colectivos conformados por personas con orientaciones e identidades de género no hegemónicas, prevalecen sectores de la comunidad universitaria (estudiantes, profesorado, personal de seguridad, etc.) anclados en concepciones tradicionales que impiden el reconocimiento y devalúan las identidades no hegemónicas, lo cual favorece al afianzamiento de prácticas de exclusión, discriminación, invisibilización y violencia contra estas personas. En las voces de los actores, estas son algunas expresiones:

Por primera vez (...) hay presencia de una persona que ingresó hace dos años y es transgénero (...). Es que llegaba con pantalonetas, shorts y blusas cortas, y un cuerpo de casi 1,75 metros, de $80 \mathrm{~kg}$ y contextura grande. Se veía extraño y se distingue de todo un grupo. No sabíamos cómo llamarlo, si como hombre o como mujer o simplemente decirle joven. La forma de vestir no es adecuada. Desde que empezó a hacer sus cambios hormonales, manifestó que no podía hacer actividad física. En clase, no sabía cómo dirigirme a esta persona y terminé llamándole "mira, tú, qué dices". Este 
personaje tuvo posturas carnavalescas y esto impactó mucho en la sede. (Docente, sede regional Tuluá, acta del grupo focal, 2018)

Las actitudes discriminatorias pueden verse más hacia mujeres trans, debido a que para las personas puede ser más sorprendente debido a la transformación física. En las inducciones, al inicio de la carrera, también vi una chica que estaba realizando su proceso de cambio de sexo, a quien varios estudiantes de varios programas académicos rechazaban con algunas actitudes. (Estudiante, sede Cali, acta del grupo focal, 2017)

Comportamientos como los narrados revelan prácticas y mecanismos a través de los cuales se invisibilizan, valoran negativamente y excluyen personas por su identidad de género, que no se sustenta en la lógica binaria, entendida como

un principio organizador del orden de relaciones sociales, política, institucional y culturalmente reproducido, que hace de la heterosexualidad reproductiva el parámetro desde el cual juzgar (aceptar, condenar) la inmensa variedad de prácticas, identidades y relaciones sexuales, afectivas y amorosas existentes: lesbianas y gais que, con sus especificidades, se apartan del patrón de heterosexualidad; las y los trans cuya identidad y expresión de género cuestionan de hecho los cánones binarios; la emergencia de las reivindicaciones intersex, que muestra hasta qué punto género y biología se entremezclan -produciendo sufrimiento evitable-. (Pecheny; Figari; Jones, 2008: 14)

Dichas prácticas pueden ser interrogadas desde un marco interpretativo en el que la construcción social del género no se asume como una posibilidad y, por lo tanto, impera una lógica de construcción identitaria estática y totalmente anclada a una determinación asumida como natural, el sexo.

Así como en los espacios de clase hay invisibilización, agresión y desconocimiento, esto también ocurre en el tránsito por las porterías de la universidad, cuando en ocasiones se interpela a las personas con identidades no hegemónicas porque su expresión de género no corresponde con el nombre que aparece en el carné que se porta, y la o lo acredita como estudiante de la universidad. En estos casos, generalmente se recurre a la negación de la identidad trans, la cual se asume como negativa. Los baños son otro lugar de agresión para los(as) estudiantes trans, porque el uso de estos no se corresponde con las expectativas de sus usuarios habituales. En estos espacios las personas trans suelen ser agredidas, intimidadas, sacadas y desvaloradas.

Si bien las personas trans reciben de manera abierta y directa las reacciones de rechazo por parte de las personas que les rodean en el entorno universitario, también las personas homosexuales se ven sometidas a prácticas estigmatizantes que califican sus comportamientos con expresiones negativas y culpabilizantes, que 
terminan aislándoles y propiciando múltiples discriminaciones. La homosexualidad (gais, lesbianas), así como las identidades de género no normativas (trans) aparecen ligadas a un comportamiento proscrito e indeseado, al tiempo que se señala a la persona como anormal e inapropiada. De acuerdo con Kristeva (1988), las personas con orientaciones sexuales e identidades de género no hegemónicas suelen ser radicalmente excluidas. En consecuencia, este comportamiento no debe ser manifestado públicamente; por el contrario, debe ocultarse o camuflarse, porque cuando se evidencia genera disturbio. La percepción sobre este asunto en uno de los grupos focales así lo ilustra:

En el 2015 un estudiante se destapó en Tecnología Electrónica y se hizo recocha y matoneo, ese estudiante desertó. Cuando se supo de la condición de ese estudiante, que era homosexual, se bloqueó la comunicación con él. Incluso el profesor dijo: “Uy, tan goloso". (Estudiante, sede regional Yumbo, grupo focal, 2018)

La han llamado lesbiana porque tiene un comportamiento diferente al que debería ser, la gente habla sin conocerla. Para ella el que a una persona le gusta su hombre o su mujer no debería ser algo que le importe a los que no están dentro de esa relación. (Estudiante, sede regional Buenaventura, grupo focal, 2018)

Así, se evidencia cómo en la convivencia cercana con estas personas emergen tensiones, algunas de las cuales se expresan en forma de divisiones dentro del grupo, en torno a las posiciones de sus integrantes frente a las diversidades. Mientras unos defienden y se identifican con quienes han asumido su orientación sexual e identidad de género de manera abierta, otros no comparten las prácticas y actitudes de estas personas en clase. A su vez, el docente describe al grupo que defiende a estas personas con el término identidad marcada, porque

usan piercing, son rockeros, son los que forman el grupo que defiende al chico gay, hacen empoderamiento para defender su diversidad, los cuales chocan abiertamente con el otro grupo, en el extremo del salón, que se muestran cansados con esa personalidad histriónica que no deja que pueda pasar desapercibido. (Docente, sede regional Santander de Quilichao, comunicación personal, 2018)

Este testimonio no solo ilustra el costo de salir del clóset y de hacerse a un lugar de reconocimiento dentro del espacio académico, sino que también deja ver cómo se convive con la diversidad, dando lugar a la llamada tolerancia, entendida como "la capacidad cívica y política de las personas de respetar 'la libertad del otro, sus maneras de pensar y de vivir, pero ella significa al mismo tiempo la presencia del 
otro a regañadientes, la necesidad de soportarlo o simplemente dejarlo subsistir'” (Pecheny, como se citó en Pecheny et al., 2008: 54).

Por otra parte, la normalidad, como un elemento imperceptible en el aula, evidencia, de acuerdo con Britzman (2002:198), "la manera inapropiada como la educación ignora las complejidades y los conflictos de la civilización”. La normalización de situaciones de hostilidad permanente que van desde la exclusión o el rechazo asociado con dejar de lado, ignorar, no dejar participar o no hablar con una persona, hasta la violencia verbal o física que incluye burlas insultos, ridiculización, motes, intimidación, acoso a través de internet y otras tecnologías de la información y la comunicación, la amenaza y la agresión física/sexual, configuran lo que se ha llamado como homofobia (Pichardo; De Stefano; Faure; Sáenz; Williams, 2015). Demostrando así, una vez más, que el problema no son las orientaciones sexuales o las identidades de género no hegemónicas, sino la homolesbotransfobia que caracteriza a las sociedades occidentales, y se refleja en la institucionalidad universitaria pese a la pretendida apertura, por lo cual debe abordarse desde sus diferentes dimensiones.

La percepción sobre la aceptación que circula a través del discurso asumido por docentes y estudiantes de la comunidad universitaria, se revela a partir de las expresiones y opiniones:

Si bien hay aceptación no hay inclusión, [expresando que] aún se perciben sesgos por parte de estudiantes y docentes que con dificultad reconocen estas otras formas de orientación sexual no hegemónica. Explica que si bien conviven como estudiantes que comparten un mismo espacio, no se sienten plenamente libres para expresar sus sentimientos, ni tampoco sienten un reconocimiento completo. (Docente, sede regional Santander de Quilichao, acta del grupo focal, 2018)

Frente a las prácticas y relaciones de género entre estudiantes, hay distanciamiento entre su discurso y sus conductas, pues mientras el primero tiende a ser incluyente y democrático, lo segundo no tanto. (Estudiante, sede Cali, acta del grupo focal, 2017)

En la universidad hay mente abierta y las personas aceptan a este tipo de personas (...). En nuestro programa académico los/as estudiantes valoramos los compañeros "más por lo que es la persona, que por su orientación sexual”. (Estudiante, sede regional Buga, acta del grupo focal, 2018)

No se reconoce resistencia a la interrelación, se tiene respeto, no se discrimina, todas las personas tienen los mismos derechos. (Estudiante, sede regional Caicedonia, acta del grupo focal, 2018)

En Contaduría se ven tendencias de algunos estudiantes, por sus maneras de hablar, de moverse, pero no se toca el tema, se asumen como otro estudiante más. (Docente, sede regional Tuluá, grupo focal, 2018) 
Se ven más hombres que mujeres y se asumen como un igual, tienen iguales derechos. No hay discriminación pero se requiere que las personas con orientación sexual diversa se sientan seguras de expresar tal como son y de ser respetadas y reconocidas y se puedan organizar. (Estudiante, sede regional Palmira, grupo focal, 2018)

Como señalan estas narraciones, en la cotidianidad universitaria las expresiones de aceptación, trato normal e igualitario, y no discriminación, transitan a la par con las bromas, agresiones y discriminaciones sobre la orientación sexual o identidad de género. De acuerdo con las percepciones de las(os) narradoras(es), estas son permitidas por las personas a las que se dirigen. No obstante, quienes son agredidos(as) expresan que el sentimiento de avasallamiento es tan demoledor que ni siquiera hay la posibilidad de respuesta.

En las relaciones interpersonales, especialmente en el caso de docentes, perciben hipocresía, dado que, si bien reciben un trato cordial de sus pares, hablan mal a sus espaldas. Entre estudiantes, la situación no es muy diferente en tanto señalan que los aceptan, pero a pesar de eso circulan rumores sobre su vida personal.

Cuando se trata de profesores diversos, una estudiante de la Facultad de Ciencias Naturales y Exactas manifiesta que la discriminación hacia un profesor gay de la facultad se da a través de burlas relacionadas con su desempeño como docente, mas no con su orientación sexual; incluso señala que en la facultad hay otro profesor gay a quien no se le hacen burlas porque su desempeño es mejor que el del primero. Tiene prestigio académico. (Estudiante, sede Cali, acta del grupo focal, 2017)

Se observa también que la aceptación de las personas con orientación sexual no hegemónica depende de la identidad de género de quien juzga y de quien es juzgado, pero también del intercambio simbólico que implica el prestigio académico. En este sentido, se encuentra que a los hombres les queda más fácil admitir la homosexualidad en las mujeres que en los hombres; lo contrario sucede con las identidades de género, donde las mujeres trans son fuertemente discriminadas en el campus y se expresa desde el no reconocimiento hasta la agresión. La discriminación es mayor si son mujeres trans negras, lo que sugiere que hay menor agresión a las mujeres trans blanco-mestizas, y que hay un factor interviniente fundamental: el prestigio académico.

Somos más tolerantes con las mujeres lesbianas que con los hombres gay (...). Pareciera que en el género femenino aceptamos y convivimos con la diversidad, pero cuando la expresión es de un hombre homosexual, con orientación femenina, no somos tan tolerantes en la convivencia. Es más fácil ver besar a dos mujeres que a dos hombres 
(...). Hay rechazo o prevención, con los hombres porque se asocia con enfermedad y prostitución. (Docente, sede regional Santander de Quilichao, grupo focal, 2018)

Los hombres son más intolerantes con este tipo de personas, porque las personas con orientación sexual diversa se identifican más con las mujeres. El padre de mi hija es homosexual y eso al principio me dio duro, pero ya no (...). Los de administración y contaduría, los hombres son los que agreden más a este tipo de personas. (Estudiante, sede regional Santander de Quilichao, grupo focal, 2018)

Al respecto, en un estudio sobre la construcción de las masculinidades realizado con hombres de diferentes clases sociales en tres ciudades peruanas, Fuller (2001) encontró que el contacto de los varones con homosexuales continúa siendo considerado como contaminante. Estas prácticas se admiten únicamente en los espacios asociados al cuerpo y sus placeres. "La masculinidad aparece como un valor importante para los varones de sectores populares" (Fuller, 2001: 71). En contraste, lo que arroja la investigación en la Universidad del Valle es que la masculinidad es un valor muy importante para ellos y de ahí el rechazo y estigma a las mujeres trans, el cual se acentúa en intersección con la pertenencia étnico-racial. Lo anterior puede ser entendido como el resultado de la presión social ejercida por un orden de género binario y heteropatriarcal blanco-mestizo, que además sitúa a las personas homosexuales y a las personas trans desde posiciones de invalidación y menosprecio, y en consecuencia los sujetos deben apegarse a ciertas pautas sociales y culturales para no ser discriminados(as) o violentados(as).

Badinter (1992), por su parte, distingue tres ejes en cuyo trayecto se construye la identidad masculina: no ser mujer, no ser bebé y no ser homosexual, de ahí que, para validar su masculinidad, los hombres deben reiterar y convencer al escrutinio social de que no es ninguna de las anteriores. En este sentido, es importante resaltar, por un lado, que la homofobia, expresada por los hombres principalmente, se deriva de una lógica heteronormativa, y constituye una postura social en contra de las diferentes expresiones de la orientación sexual que no puede ser pensada como un comportamiento individual y aislado (Butler, 2002; Granados, 2002; Guasch, 2007). En la voz de las(os) estudiantes:

En Meléndez lo que hemos visto son manadas de personas con orientación sexual diversa. En la sede (...) no se ve, somos más sanos, no conocemos aquí ni gais, ni lesbianas. Otro estudiante expresó que en la sede se han visto casos de hombres y de mujeres. También el grupo comentó que en las mujeres se disimula más. En los hombres es más difícil porque después quien los vea lo puede considerar como que le 
gustan los hombres. También que si tiene amiga lesbiana, puede ser que de pronto la coquetea. Por ejemplo, el caso de una estudiante lesbiana que estaba en el equipo de fútbol, el entrenador la retiró. (Estudiantes, sede regional Yumbo, grupo focal, 2018)

En este contexto, el discurso de aceptación e igualdad de trato no es suficiente, y riñe con el propósito de reconocer a las personas con orientación sexual y de identidad de género no hegemónica. Por el contrario, deshistoriza las identidades de género al sustraer el carácter político de las mismas (Lo-Re, 2014), en tanto la persona pasa como igual a todos y se invisibiliza, se normaliza la violencia contra estas personas a través del chiste o tiene que condicionarse, ajustarse y ocultarse para evitar el hostigamiento. Como lo ilustran los siguientes testimonios, se construye una armonía sujeta a ciertas condiciones relacionadas con la restricción de las conductas y de las expresiones erótico-afectivas de estas personas en el campus, llevándoles a mostrar pasividad ante las hostilidades. La forma de vestirse y de expresarse debe estar alineada con los estereotipos y el orden de género imperante, lo cual conlleva a ocultar estas manifestaciones y, por tanto, evita que los actores involucrados en la cotidianidad universitaria se pregunten por el carácter ideológico y moralizante que hace de la heteronormatividad la ideología de la exclusión de la diversidad sexual (Pecheny et al., 2008).

Un estudiante expresa en el grupo focal que es gay y que el semestre pasado él tenía novio, se besaba con él en la universidad. Los de tecnología (Logística Portuaria) le decían cosas en modo de burla: "Mario, el marica”, o "este niño... [mejor] esta niña", sin ni siquiera conocerlo. (Estudiante, sede regional Buenaventura, grupo focal, 2018)

Al interior de la universidad sí ha notado discriminación hacia las personas con diversidades sexuales, en actitudes como: evitar los lugares en los que ellos están, excusando tal actitud en que "ay, no, es que no sé cómo comportarme frente a ellos"; y expresiones verbales como "yo te respeto, pero no me junto con vos". (Estudiante, sede Cali, acta del grupo focal, 2017)

Al respecto, Andrés Castelar y Jenny Lozano (2018) señalan que estas conductas imponen la privatización de las expresiones eróticas no hegemónicas, el silencio del deseo y la pseudotolerancia, lo cual es ilustrado por las(os) estudiantes de la siguiente manera:

Cuando se tiene una pareja homo en la universidad, no se pueden expresar el afecto libremente en el público porque hay malas miradas, comentarios y chistecitos. Pero si es una pareja hetero nadie puede decir nada, ellos se creen que pueden mostrarse el 
afecto en público porque son hombre y mujer. (Estudiante, sede regional Buenaventura, grupo focal, 2018)

Estas percepciones asociadas con las dinámicas que toman las expresiones de la diversidad sexual en la universidad no solo encierran una concepción binaria y estática de la sexualidad y de las identidades de género, sino que permiten que el estigma opere como práctica reguladora, generando culpa, autocontrol y autovigilancia de la sexualidad y del género (Serrato; Balbuena, 2015). Desde este sistema sexual jerárquico, cuerpos y sexualidades son marcados y regulados. Por ello, la mayoría de estudiantes con orientación sexual y de género no hegemónica optan por ocultar su condición en algunos espacios, y mostrarla en otros, donde expresar abiertamente su orientación sexual o identidad de género no les represente ser excluidos. Permanentemente, estas personas deben decidir entre el ocultamiento y el afrontamiento, entendiendo que este último implica soportar el señalamiento y las hostilidades provenientes de sus pares en el marco de una cultura institucional que oscila entre la apertura, la aceptación a lo no hegemónico y el simbolismo heteronormativo hegemónico, que se muestra tolerante a prácticas de marcaje como el chisme, burla, risas, comentarios, miradas, señalamientos, insultos verbales o agresiones físicas, y rechazo (Segura, 2015).

He tenido compañeros que se han reconocido como tal, pero otros no lo han hecho porque temen al rechazo que se genera por parte de las personas. (Estudiante, sede regional Buga, grupo focal, 2018)

En cuanto a las orientaciones sexuales no es muy visible por el camuflaje en el colectivo; pero se puede detectar cuando se tienen más cerca como en las asesorías ya que se tiene trabajo en parejas o pequeños grupos. También, cuando se acercan a nosotros y nos comentan. Por eso las orientaciones sexuales de estudiantes no se hacen tan visibles porque tienen temor al rechazo, porque conocen de casos de bullying a estas personas. Por ejemplo, en Tecnología Agroambiental, comenta una docente, en una salida o en un trabajo de campo hacen burlas, comentarios y se comportan delante de la profesora de un modo y cuando ella no está se hacen de otro modo. (Docente, sede regional Palmira, grupo focal, 2018)

El simbolismo propio del orden heteronormativo y hegemónico se expresa en las áreas de saber que hacen presencia en la universidad. En los campos disciplinares considerados socialmente de mayor prestigio académico (ingenierías, ciencias exactas, salud, entre otros) se observa que hay menor aceptación a las personas con orientación sexual e identidad de género no hegemónicas, en contraste con 
campos del saber que se consideran con menor prestigio social (humanidades o ciencias sociales), en los que las mujeres y las personas con orientación sexual e identidad de género no hegemónica tienen mayor aceptación e incluso alcanzan algún reconocimiento a sus identidades y desempeño académico. Así, encontramos carreras y personas estigmatizadas por estas percepciones que se traducen en desventajas académicas y laborales con respecto a la población heterosexual que estudia carreras que no están etiquetadas de esta forma. Es así que la universidad se configura como un espacio que reproduce y naturaliza las desigualdades entre los grupos y las personas, en tanto se dan prácticas que refuerzan patrones de exclusión y discriminación (Alarcón; Toquica, 2017).

Los aspectos descritos nos llevan a plantear que son diversos los mecanismos de exclusión que en el contexto universitario afectan el reconocimiento de las personas con orientaciones sexuales e identidades de género no hegemónicas, dentro de una Institución de Educación Superior en la que también se enarbola la aceptación y tolerancia. Algunos de estos mecanismos se expresan de forma diferenciada con relación a la orientación sexual y la identidad de género. Por un lado, encontramos un conjunto de prácticas y expresiones por parte de la comunidad estudiantil hacia estas personas, que buscan naturalizar el hostigamiento permanente, ocultándolo a través de chistes, burlas o aislamiento. Por otra parte, operan mecanismos simbólicos asociados con la autorregulación y el autocontrol de las expresiones erótico-afectivas por parte de estas personas, que dan lugar a lo que llamamos una presencia condicionada en la universidad, la cual permite a quienes se ocultan construir una armonía que evite su exclusión, y a quienes asumen abiertamente su orientación sexual o identidad de género experimentar un ambiente de permanente tensión y rechazo, al tiempo que ganan visibilidad como sujetos con derechos y contribuyen a consolidar su reconocimiento como sujetos sociales y políticos.

\section{Diversidades de género y reconocimiento en la universidad}

El conjunto de interacciones cotidianas marcadas por la discriminación y la falta de reconocimiento en la universidad, algunas relatadas hasta aquí y presentes tanto en estudiantes como en docentes con sus matices y singularidades, ponen en tensión el discurso de apertura, aceptación y tolerancia hacia las personas con orientación sexual y de género no hegemónica. Una docente señala claramente esta situación, al expresar que, si bien hay aceptación, no hay inclusión, expresando que aún se perciben "sesgos por parte de estudiantes y docentes que con dificultad reconocen 
estas otras formas de orientación sexual no hegemónica" ${ }^{10}$. Lo anterior sugiere que estos estudiantes que comparten un mismo espacio no se sienten plenamente libres para expresar sus sentimientos ni tampoco completamente reconocidas(os).

Como se ha señalado a lo largo del texto, esta falta de reconocimiento se refleja en actitudes de rechazo encubiertas con burlas, chistes y aislamiento. Como muestran los resultados de la investigación, la exclusión y la discriminación se expresan en diferentes contextos y de variadas formas en la universidad, lo cual se constituye en una falta de reconocimiento y configura lo que Fraser (2000) aborda desde el debate sobre la justicia (redistribución-reconocimiento). Sin intención de profundizar en él, es importante señalar, a partir de los estudios revisados y de los resultados arrojados, que nos parece primordial referirnos a algunos aspectos clave de este debate para interpretar cómo la lógica heteronormativa, reforzada con la heterosexualidad impuesta a través de diversos mecanismos de discriminación, entre ellos el discurso de tolerancia", caracterizado por "la apertura, la aceptación y el trato igualitario", termina generando una injusticia que debe ser considerada desde una perspectiva que busque, de acuerdo con Fraser (2000), la recuperación y la integración, la cual no puede desconocer la opresión heterosexista, como tampoco la legitimidad moral de las reivindicaciones gais y lesbianas.

En primer lugar, de acuerdo con Castelar (2010), la existencia de una legislación que reconozca a estas personas como sujetos sexuales y les garantice sus derechos no es suficiente para su reconocimiento pleno, dado que, como señala el autor, este "va más allá de la aceptación jurídica de los derechos individuales y de la redistribución y la aceptación, porque esto no ha significado reconocer al diverso/a, como un participante paritario en el concierto social" (Castelar, 2010: 208). Ello nos permite interpretar, en el caso de la universidad, que es fundamental avanzar en el reconocimiento de las diversidades sexuales y de identidad de género, más allá de lo normativo, e instaurarlo en el mundo simbólico institucional, cuestionando la generización de los campos del saber y su transmisión acrítica. En términos de Nancy Fraser (200o), valorando las identidades injustamente devaluadas cuando el otro por su orientación sexual o identidad de género es inferiorizado, menospreciado o considerado inapropiado en determinadas disciplinas, que debe ocultarse en determinadas situaciones o expresarse bajo ciertos parámetros definidos por el orden de género heteronormativo. Desde esta perspectiva, y de acuerdo con Fraser (2000), la falta de reconocimiento es una relación social institucionalizada y no un

10. Docente, sede regional Santander de Quilichao, acta del grupo focal, 2018.

11. Utilizamos este concepto porque es una expresión de los(as) actoras(es) participantes en la investigación. 
estado psicológico. El estado de cosas que hemos descrito hasta aquí se concibe como una falta de reconocimiento

que no equivale simplemente a ser desahuciada como una persona enferma, ser infravalorado o recibir un trato despreciativo en función de las actitudes conscientes o creencias de otras personas, falta de reconocimiento en este caso, equivale a no ver reconocido el propio status de interlocutor/a pleno/a en la interacción social y verse impedido/a participar en igualdad de condiciones en la vida social, no como consecuencia de una desigualdad en la distribución (como, por ejemplo, verse impedida a recibir una parte justa de los recursos o de los "bienes básicos"), sino, por el contrario, como una consecuencia de patrones de interpretación y evaluación institucionalizados que hacen que una persona no sea comparativamente merecedora de respeto o estima. (Fraser, 2000: 124-125)

Siguiendo los planteamientos de Fraser (2000), si bien gais y lesbianas sufren graves injusticias económicas, estas se derivan del orden de estatus, pues la institucionalización de normas heterosexistas produce una categoría de personas despreciables que afrontan prejuicios económicos a consecuencia de su estatus subordinado y, en este sentido, la falta de reconocimiento constituye una injusticia fundamental que, implique o no una redistribución, tiene consecuencias políticas, por lo cual debe ser legitimada y remediada de tal manera. De lo que se trata es de elaborar un enfoque integrado que englobe y armonice ambas dimensiones de la justicia social: redistribución y reconocimiento.

De acuerdo con Fraser (2000), el reconocimiento para gais, lesbianas y las identidades de género no hegemónicas desvalorizadas implica deconstruir las normas heterosexuales y revalorizar la sexualidad y las identidades despreciadas, acordando su reconocimiento positivo, el cual debe reflejarse en una mejora de su posicionamiento político y económico en un determinado contexto. Las Instituciones de Educación Superior deben trabajar en una transformación axiológica para que se pase de la apertura y la normalización al reconocimiento de las personas con orientación sexual e identidad de género no hegemónicas. Es en este sentido que se deben formular las políticas de género para las IES.

\section{A modo de conclusión}

En la Universidad del Valle, a pesar de su poco, pero significativo avance normativo en relación con el reconocimiento de las diversidades, especialmente las de género y orientación sexual a través de las resoluciones y programas mencionados, persiste 
una gran distancia con respecto a las prácticas y relaciones que se establecen entre los distintos integrantes de la comunidad universitaria y esta población. Si bien se escuchan voces expresando aceptación, normalidad e igualdad de oportunidades para estas personas, al mismo tiempo se vivencian interacciones en diferentes ámbitos universitarios cargadas de discriminaciones, invisibilización y exclusión.

La estigmatización a la que es sometida esta población se fundamenta en una lógica heteronormativa que fortalece la heterosexualidad obligatoria. Se manifiesta a través de prejuicios, chismes, burlas, risas, comentarios, miradas, señalamientos, insultos verbales, agresiones físicas y hasta segregación; también al confinarse a las personas con orientación sexual e identidad de género no hegemónica a ciertos espacios físicos de la universidad y a carreras con características determinadas. Como señala Fraser (2000), la falta de reconocimiento de las diversidades es el resultado de la institucionalización de normas heterosexistas que producen otros menospreciados e inapropiados, en ese sentido, es necesario resaltar el papel de la universidad como espacio de producción de conocimiento, así como su potencial transformador de valores, y actitudes positivas y edificantes de la inclusión y de la democracia, aspectos desde los cuales debe avalar la confrontación sobre este tipo de asuntos.

Finalmente, los resultados del estudio nos permiten dimensionar el grado de justicia que se ha alcanzado en la universidad. Es claro que no se trata de percatarse de la diferencia que comportan las personas que integran la comunidad universitaria, sino de resignificar positivamente al otro(a) desde esa diferencia, sin verlo como alguien con quien toca convivir porque asílo establece el manual de convivencia. Se trata de verlo(a) como alguien a quien se valora y respeta, precisamente porque tiene derecho de ser y hacer diferente. En este sentido, encontramos que hay mayor resistencia de los hombres heterosexuales a aceptar y reconocer las personas homosexuales y transgénero, que de las mujeres heterosexuales a aceptar y reconocer a personas con orientaciones sexuales de género no hegemónicas. Las explicaciones al respecto se pueden ubicar en la construcción de masculinidades hegemónicas y sexualidades heteronormativas que pueden contribuir a que se considere como abyecto a quien y todo lo que se distancia de esos patrones socioculturales. También es importante examinar la misoginia como un aspecto que reconfigura la homofobia, debido a que, si la identidad masculina nace de la renuncia a lo femenino, entonces lo que causa temor es la "feminización con la que el cuerpo del varón aparece transgrediendo los ideales normativos de lo que Butler denominaría la 'cogerencia heteronormativa"” (Méndez, 2017: 679).

Desde esta perspectiva, se espera que la universidad aporte a la construcción de entornos que favorezcan la expresión y el desarrollo de esas personas cuya identidad está atravesada por alguna condición que la hace diferente, y se interrogue, de acuer- 
do con Britzman (2002:198), "cómo podría la pedagogía desarrollar un pensamiento sobre la ausencia de la normalidad" que cuestione los binarismos tan presentes en el ejercicio educativo y en los campos del saber que en esta se agencian. Igualmente, se debe resaltar la necesidad de abordar las diversidades sexuales y de género no solo como víctimas de un orden hegemónico excluyente que les discrimina y afianza las desigualdades, sino como personas con sus propias preferencias y derechos, con posibilidades de disfrutarlos y con las mismas opciones de ser aceptados y valorados en su singularidad (Honneth, 2004).

Las IES no pueden continuar en una lógica de opuestos, sino que deben apostarle a la construcción de reconocimiento de las identidades devaluadas injustamente, lo cual permitirá una transformación axiológica real y un aporte a la construcción de una sociedad realmente incluyente, razón de ser de las instituciones educativas.

\section{Referencias}

Alarcón, Blanca; Toquica, Adriana (2017). Discriminación y violencia en jóvenes universitarios en Bogotá, 2016 [Tesis de maestría]. Universidad Santo Tomás, Bogotá.

Arango, Luz Gabriela (2006). Jóvenes en la universidad: género, clase e identidad profesional. Bogotá: Siglo del Hombre/Universidad Nacional de Colombia.

Arango, Mauricio (2017). Prácticas de exclusión socialy construcción identitaria de personastransgénero en contextos universitarios [Tesis de maestría]. Universidad de Antioquia, Medellín.

Badinter, Elisabeth (1992). XY de la identidad masculina. París: Odile Jacob.

Barrancos, Dora (2007). Reseña de "La cuestión gay. Un enfoque sociológico" de Ernesto Meccia. Revista Argentina de Sociología, 5(9), 160-163. Recuperado de https://www.redalyc. org/pdf/269/26950911.pdf

Britzman, Deborah (2002). Pedagogías transgresoras y sus extrañas técnicas. En Sexualidades transgresoras. Una antología de estudios Queer (pp.197-228), coordinado por Rafael Mérida. Barcelona: Icaria.

Butler, Judith (2002). Cuerpos que importan: sobre los límites materiales y discursivos del "sexo". Buenos Aires: Paidós.

Cáceres, Carlos; Rosasco, Ana (200o). Secreto a voces. Homoerotismo masculino en Lima: culturas, identidades y salud sexual. Lima: Universidad Peruana Cayetano Heredia. 
Campo, Adalberto; Oviedo, Heidi; Herazo, Edwin (2017). Correlación entre actitud hacia homosexualidad femenina y masculina en estudiantes de Medicina. Psicogente, 2O(37), 173-181. https://doi.org/10.17081/psico.20.37.2426

Castelar, Andrés (2010). Judith Butler y el problema del reconocimiento. En Identidades colectivasy reconocimiento: razas, etnias, géneroy sexualidades (pp. 193-218), compilado por Delfín Grueso; Gabriela Castellanos. Santiago de Cali: Universidad del Valle.

Castelar, Andrés; Lozano, Jenny (2018). Reconocimiento de la diversidad sexual en la escuela: algunas paradojas. Psicología, Epistemología y Sociedad, 25, 55-79. http://dx.doi. org/10.18046/recs.i25.2220

Comisión Internacional de Juristas (2009). Orientación sexual e identidad de género y derecho internacional de los derechos humanos. Suiza: CIJ.

Curiel, Ochy (2011). Género, raza y sexualidad. Debates Contemporáneos. Recuperado de https://www.urosario.edu.co/Subsitio/Catedra-de-Estudios-Afrocolombianos/Documentos/13-Ochy-Curiel---Genero-raza-y-sexualidad-Debates-.pdf

Curiel, Ochy (2012). Prólogo. En Gaisy lesbianas en contextos universitarios de Cali Colombia (pp. 15-17), compilado por Hernando Uribe; Lorena Rodríguez; Gilberto Aristizábal. Santiago de Cali: Universidad Autónoma de Occidente.

Fernández, Sara; Hernández, Gloria; Paniagua, Ramón (2013). Violencia de género en la Universidad de Antioquia. Medellín: Universidad de Antioquia.

Figari, Carlos (2008). Identidades de género y prácticas sexuales. Heterosexualidades masculinas flexibles. En Todo sexo es político: estudios sobre sexualidades en Argentina (pp. 97-122), compilado por Mario Pecheny; Carlos Figari; Daniel Jones. Buenos Aires: Libros del Zorzal.

Fondo de Población de las Naciones Unidas (2012). Ampliando la mirada: la integración de los enfoques de género, interculturalidady derechos humanos. Santiago de Chile: UNFPA.

Fraser, Nancy (2000). Heterosexismo, falta de reconocimiento y capitalismo: una respuesta a Judith Butler. New Left Review, 2, 123-136. Recuperado de https://newleftreview.es/issues/2/ articles/nancy-fraser-heterosexismo-falta-de-reconcimiento-y-capitalismo-una-respuesta-a-judith-butler.pdf

Fuller, Norma (2001). Masculinidades. Cambiosy permanencias: varones de Cuzco, Iquitosy Lima. Lima: Pontificia Universidad Católica del Perú.

Gilbert, Miqqi (2011). Derrotando el bigenerismo: cambiando supuestos de género en el siglo XXI. Nomadías, 13, 103-128. https://doi.org/10.5354/0719-0905.2011.15288

Granados, José (2002). Orden sexual y alteridad: homofobia masculina en el espejo. Nueva Antropología, 61, 79-97. 
Guasch, Oscar (2007). La crisis de la heterosexualidad. Barcelona: Laertes.

Guerrero, Ana; Zambrano, Alexander (2013). Prejuicios y estereotipos en estudiantes de la Universidad de Nariño. Plumilla Educativa, 12(2), 71-92.

Honneth, Axel (2004). Recognition and Justice: Outline of a Plural Theory of Justice. Acta Sociológica, 47(4), 351-364. https://doi.org/10.1177/0001699304048668

Instituto Nacional Contra la Discriminación, Xenofobia y el Racismo (2016). Diversidad sexual $y$ derechos humanos: sexualidades libres de violencia y discriminación. Buenos Aires: INADI.

Kimmel, Michael (1997). Homofobia, temor, vergüenza y silencio en la identidad masculina. Ediciones de las Mujeres, 24, 49-62.

Kristeva, Julia (1988). Poderes de la perversión. Ensayo sobre Louis-Ferdinand Céline. Madrid: Siglo XXI.

Lo-Re, Silvina (2014). Heteronormatividad, escuela, identidad sexual y Estado. Recuperado de https://diariofemenino.com.ar/heteronormatividad-escuela-identidad-sexual-y-estado/

López, Yessica; Martínez, Angie; Negrete, Yiseth; Paternina, Saira (2016). Factores asociados a la homofobia en estudiantes de enfermería en las universidades de la ciudad de Cartagena (Trabajo de grado). Universidad de Cartagena, Cartagena.

Lugones, María (2008). Colonialidad y género. Tabula Rasa, 9, 73-101.

Martínez, Lola (2018). Disidencias sexuales y corporales: articulaciones, rupturas y mutaciones. Psicoperspectivas, 17(1).https://doi.org/10.5027/psicoperspectivas-vol17-issue1-fulltext-1141

Méndez, Manuel (2017). Reflexiones críticas sobre homofobia, educación y diversidad sexual. Educação or Realidade, 42(2), 673-686.

Moore, Henrietta (1991). Antropología y feminismo. Madrid: Cátedra.

Munévar, Dora (2013). Interseccionalidad y otras nociones. En La interseccionalidad en debate. Actas del Congreso Internacional "Indicadores Interseccionales y Medidas de Inclusión Social en Instituciones de Educación Superior” (pp.56-65), editado por Marta Zapata; Sabina García; Jennifer Chan. Berlín: Freie Universität Berlin. Recuperado de http://www.miseal.org/ index.php/publicaciones/36- documentacion/publicaciones/147

Organización Mundial de la Salud (2015). Sexual Health, Human Rights and the Law. Génova: OMS.

Pecheny, Mario; Figari, Carlos; Jones, Daniel (2008). Todo sexo es político. Estudios sobre sexualidades en Argentina. Buenos Aires: Libros del Zorzal.

Pichardo, José; De Stefano, Matías; Faure, Julia; Sáenz, Marina; Williams, Jennifer (2015). Abrazar la diversidad: propuestas para una educación libre de acoso homofóbico y transfóbico. Madrid: Instituto de la Mujer y para la Igualdad de Oportunidades. 
Rich, Adrienne (1980). Compulsory Heterosexuality and Lesbian Existence. Signs, 5(4), 631-66o. Scott, Joan (2008). Género e historia. México: FCE.

Segura, José (2015). “¿Dime con quién andas y te diré quién eres?”. Sexualidad, violencia y vida cotidiana en Puerto López. Virajes, 17(2), 79-94.

Serrano, José; Viveros, Mara (2006). Regímenes de ordenamiento social y representaciones de diferencia en Colombia contemporánea. En Sexualidad, estigma y derechos humanos (pp. 96-112), editado por Carlos Cáceres; Gloria Careaga; Tim Frasca; Mario Pecheny. Lima: FASPA/UPCH.

Serrato, Abraham; Balbuena, Raúl (2015). Calladito y en la oscuridad. Heteronormatividad y clóset, los recursos de la biopolítica. Culturales, 3(2), 151-18o.

Serret, Estela (2011). Hacia una redefinición de las identidades de género. GénEros, 9, 71-97.

Uribe, Hernando (2012). Gays y lesbianas en contextos universitarios de Cali, Colombia. Cali: Universidad Autónoma de Occidente.

Vergel, Mawency; Martínez, José; Zafra, Liliana (2016). Factores asociados al bullying en instituciones de educación superior. Revista Criminalidad, 58(2), 197-208.

Viveros, Mara (2016). La interseccionalidad: una aproximación situada a la dominación. Debate Feminista, 52, 1-17. http://dx.doi.org/10.1016/j.df.2016.09.005

Wittig, Monique (1992). El pensamiento heterosexualy otros ensayos. Madrid: Egales.

Zambrano, Gloria; Escalante, Harrinson (2013). Grado de homofobia en estudiantes de enfermería de una universidad pública en Colombia. Ciencia y Cuidado, 1o(2), 115-126. 